\title{
Image Quality Improvement of Low-Resolution Camera Using Data Fusion Technique
}

\author{
S. A. Quadri and Othman Sidek
}

\begin{abstract}
Data fusion techniques can combine data from multiple information sources to achieve improved accuracies and enhanced precision. The study aims at evaluation of data fusion technique using frequency distribution analysis. Frequency distribution analysis is carried out using Matlab and Simulink, which summarizes image statistics and represents them in histogram. Energy associated with the image is studied using singular value decomposition (SVD) analysis. Considering images as input data, data fusion is carried out by using commercial available software. It is shown that the quality of image after fusion is substantially improved. By image processing and analyzing the results, it is tried to affirm that synergy is obtained using data fusion technique.
\end{abstract}

Index Terms-Data fusion, image processing, frequency distribution analysis, singular value decomposition

\section{INTRODUCTION}

Data fusion deals with the synergistic combination of information made available by different measurement sensors, information sources, and decision makers. Thus, sensor fusion is concerned with distributed detection, sensor registration, data association, state estimation, target identification, decision fusion, user interface and database management. A group of many incomplete sets of data from many sensors may be fused and lead to useful and unambiguous declarations. This effect obtained from the fusion is called synergy [1]-[2].

Image fusion is defined as the process of combining multiple input images into a single composite image. The aim is to create from the collection of input images a single output image, which contains a better description of the scene than the one provided by any of the individual input images. The output image should therefore be more useful for human visual perception or for machine perception.

The principal motivation for image fusion is to improve the quality of the information contained in the output image in a process known as synergy. A study of existing image fusion techniques and applications shows that image fusion can provide us with an output image with an improved quality. The major benefits of image fusion include extended range of operation, extended spatial and temporal overage, reduced uncertainty, increased reliability, robust system performance, and compact representation of information [3].

The image fusion synergy process can be illustrated by following three examples. The first example, multiple camera surveillance systems (MCSS) [4] that deals with input images,

Manuscript received September 12, 2012; revised October 23, 2012

The authors are with Collaborative Micro-electronic Design Excellence Centre (CEDEC), Universiti Sains Malaysia, Engineering Campus, 14300, Malaysia (e-mail: reachquadri@yahoo.com,othman@cedec.usm.my). which are captured by the same camera at different times. The next example, multispectral bilateral video fusion (MBVF) [5], that deals with input images captured by different cameras at the same time, while the third example, color image segmentation (CIS) [6], which deals with the input images which are derived from the same base image and which are processed differently. In all the three cases, data fusion has improved the quality of images for better inference and decision-making.

Application of data fusion technique to improve quality of an image for better understanding and inference is an active research area [7]-[9]. Low-cost feature of low-resolution optical sensors has attracted its usage in many applications [10]-[13]. The paper presents a simple demonstration of image fusion using commercial available software. The quality of image captured from low-resolution image sensor (mobile camera of $2 \mathrm{M}$ pixel), after fusion seems to be improved, which is obviously perceptible as shown in Fig.1. Feature level analysis is carried on individual images and fused image using Matlab.

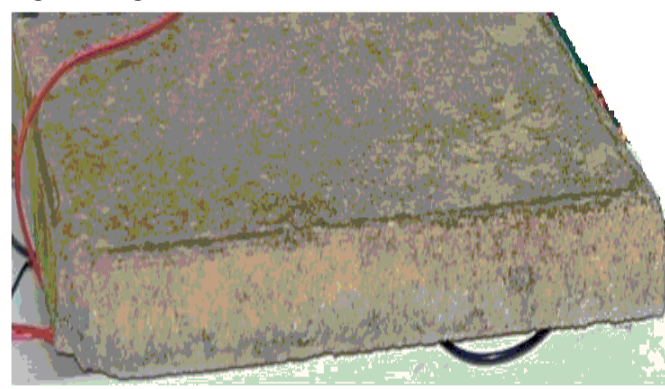

Fig. 1a. First image of slab captured using LROD (LROD-low-resolution optical device)

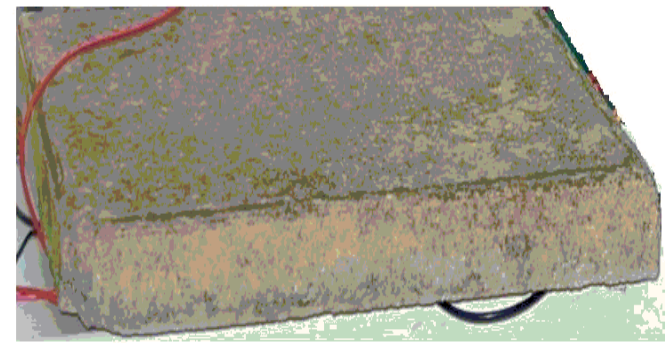

Fig. 1b. Second image of slab captured using LROD

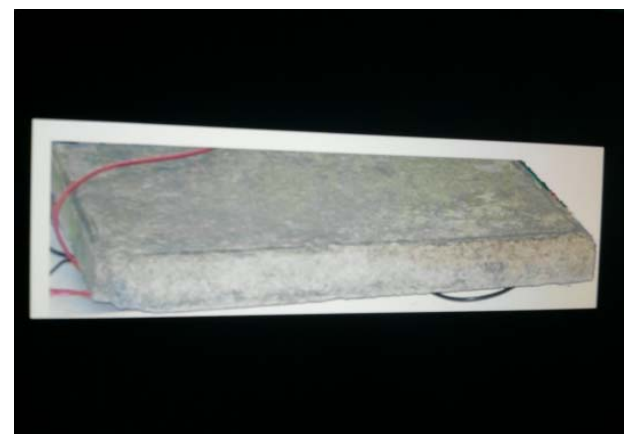

Fig. 1c. Image after fusion 


\section{DATA FUSION}

Traditionally, the input images are captured by the same camera at different times or are captured by different cameras at the same time. In this experiment, same low costing mobile camera of 2-Mega pixel resolution is used sequentially to capture the images of a concrete slab. Any mobile/digital camera records the time-and space-varying light intensity information reflected and emitted from the object. The schematic diagram of image fusion is shown in Fig. 2.

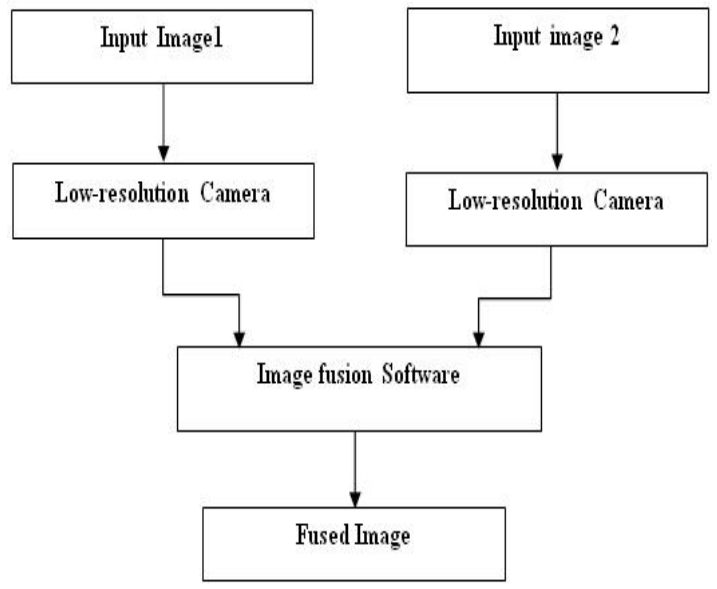

Fig. 2. Schematic diagram of image fusion

DreamFusion [14], commercially available software is used for fusing two images of concrete slabs as shown in Fig. 3 . The software takes care of combining relevant information in two or more images. It reads in a set of images and combines them into a single highly informative image. The input and output formats for the images include a wide range of formats, including tif, jpg, gif, png, bmp, pgm etc. The software manages automatic image alignment, proper sampling and noise reduction in the process of fusion.

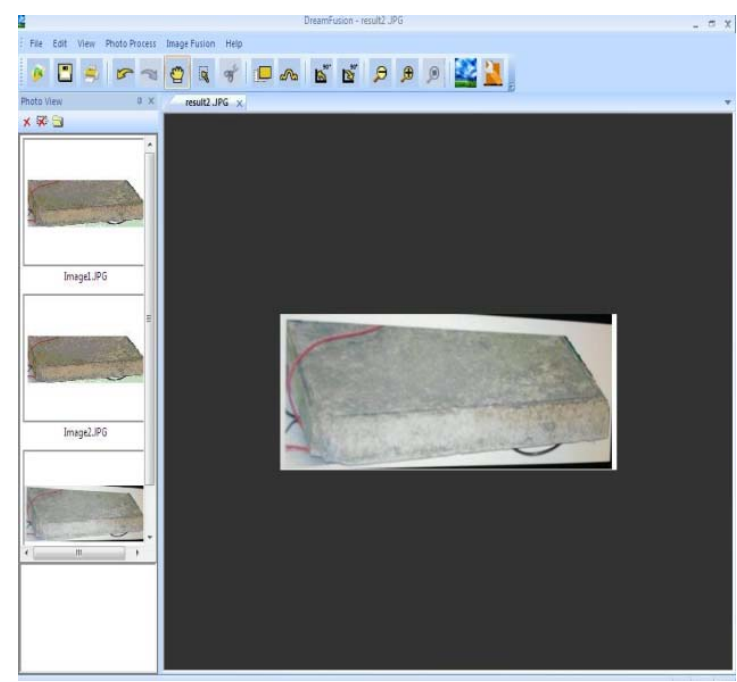

Fig. 3. Screen shot of dream fusion software

\section{IMAGE PROCESSING}

The image is processed using Matlab Image processing toolbox and Simulink [15]. RGB images are converted to matrices as an initial step for processing. The histogram block computes the frequency distribution of the elements in each input image by sorting the elements into a specified number of discrete bins. We can use the histogram block to calculate the histogram of the R, G, and/or B values in an image. It computes the frequency distribution of the elements in a vector input, of the elements in each channel of a frame-based matrix input. The running histogram parameter selects between basic operations and running operation. The histogram block accepts real and complex fixed-point and floating-point inputs.

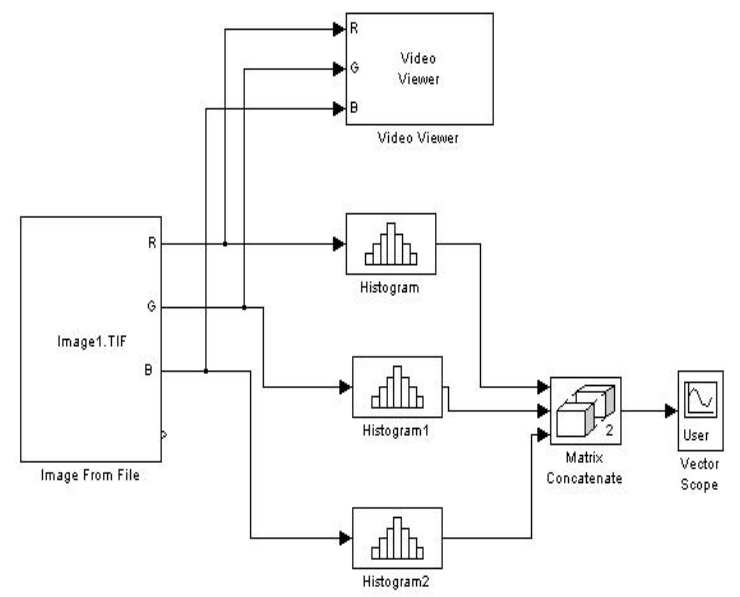

Fig. 4a. Simulink computation of image 1

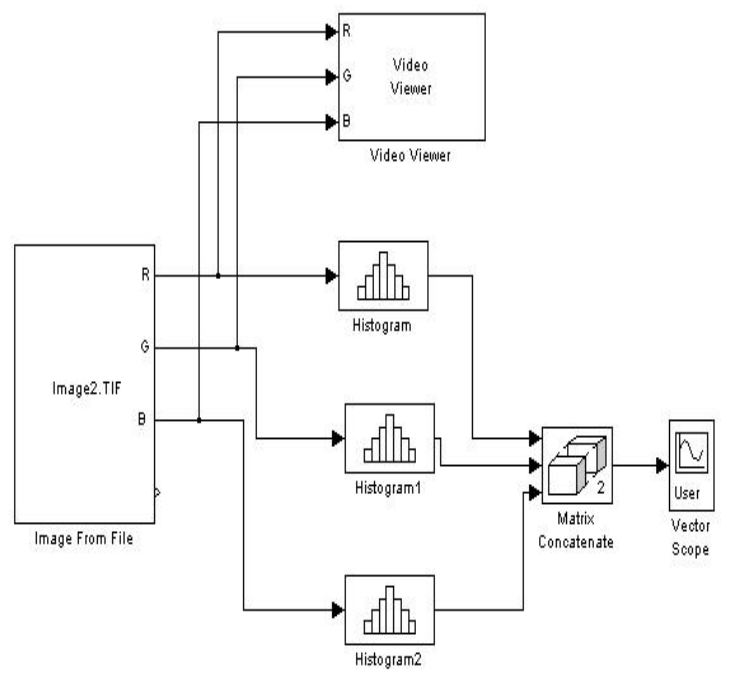

Fig. 4b. Simulink computation of image2

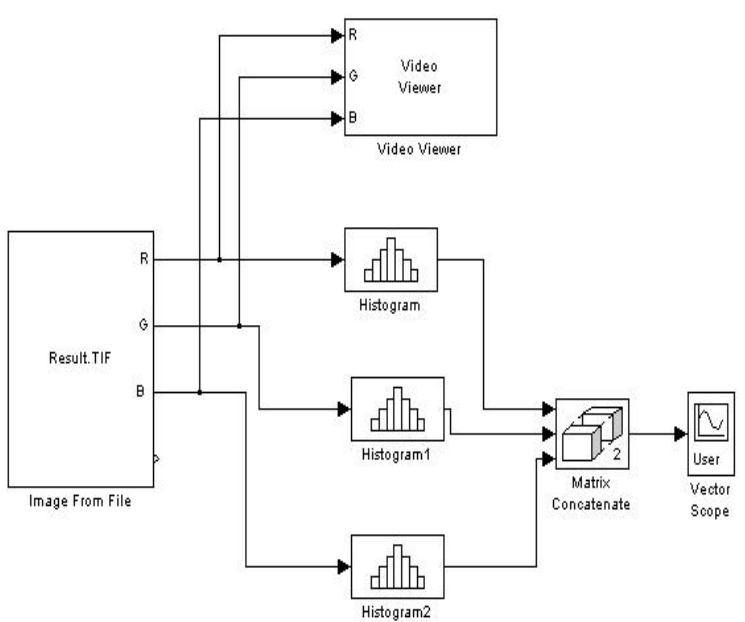

Fig. 4c. Simulink computation of fused image

The block distributes the elements of the input into the number of discrete bins specified by the number of bins parameter, $\mathrm{n}$.

$\mathrm{y}=$ hist $(\mathrm{u}, \mathrm{n}) \quad \%$ Equivalent MATLAB code

The histogram value for a given bin represents the 
frequency of occurrence of the input values bracketed by that bin. The histogram of respective image is computed using Simulink as shown in Fig. 4 a, b, c.

\section{RESUlTS AND DiscusSiON}

As shown in Fig.5. a, b, c, the frequency distribution of image 1 and image 2 are almost identical which is also an indicating factor that the quality of both the images are approximately same. After the fusion of two images, the frequency distribution of resulting image is quite different, distinct over shoot spike is shown in the Fig.5c. The same histograms are smoothed and redrawn to log based graph in Fig.6. a, b c. The histogram bins are represented in $\mathrm{X}$-axis while frequency in Y-axis.

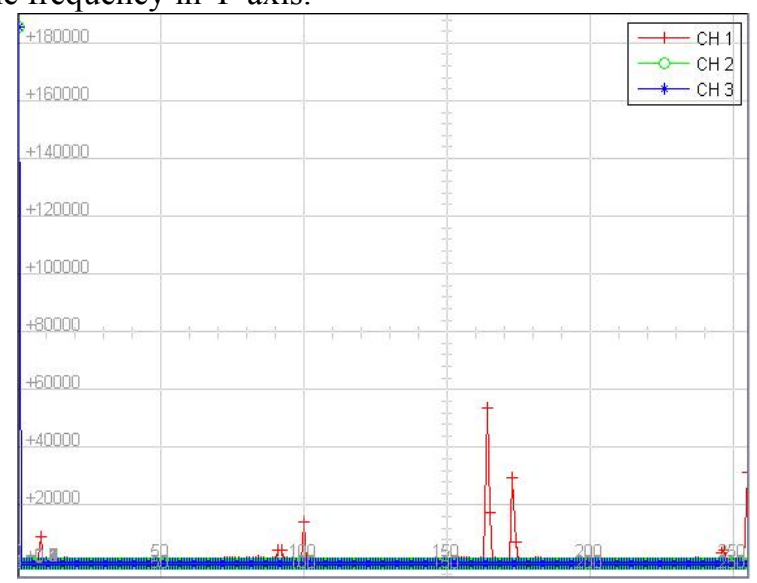

Fig. 5a. Histogram output of image1

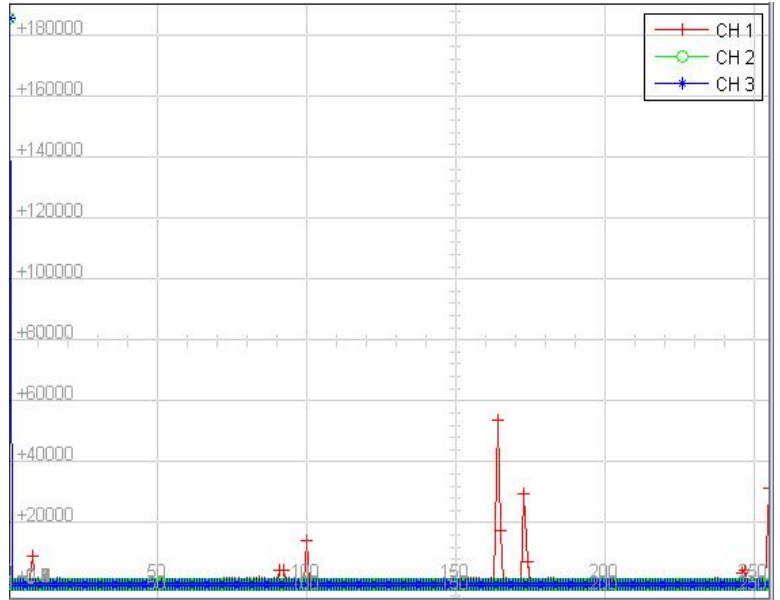

Fig. 5b. Histogram output of image2

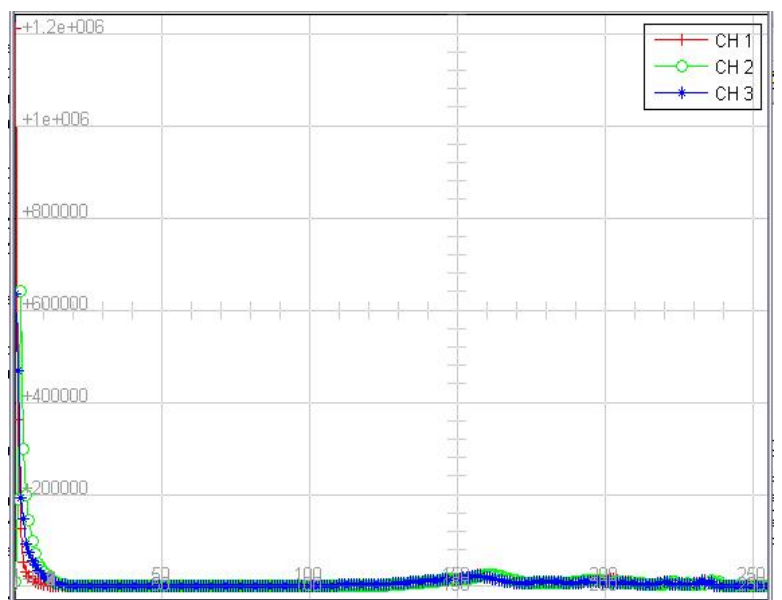

Fig. 5c. Histogram output of fused image
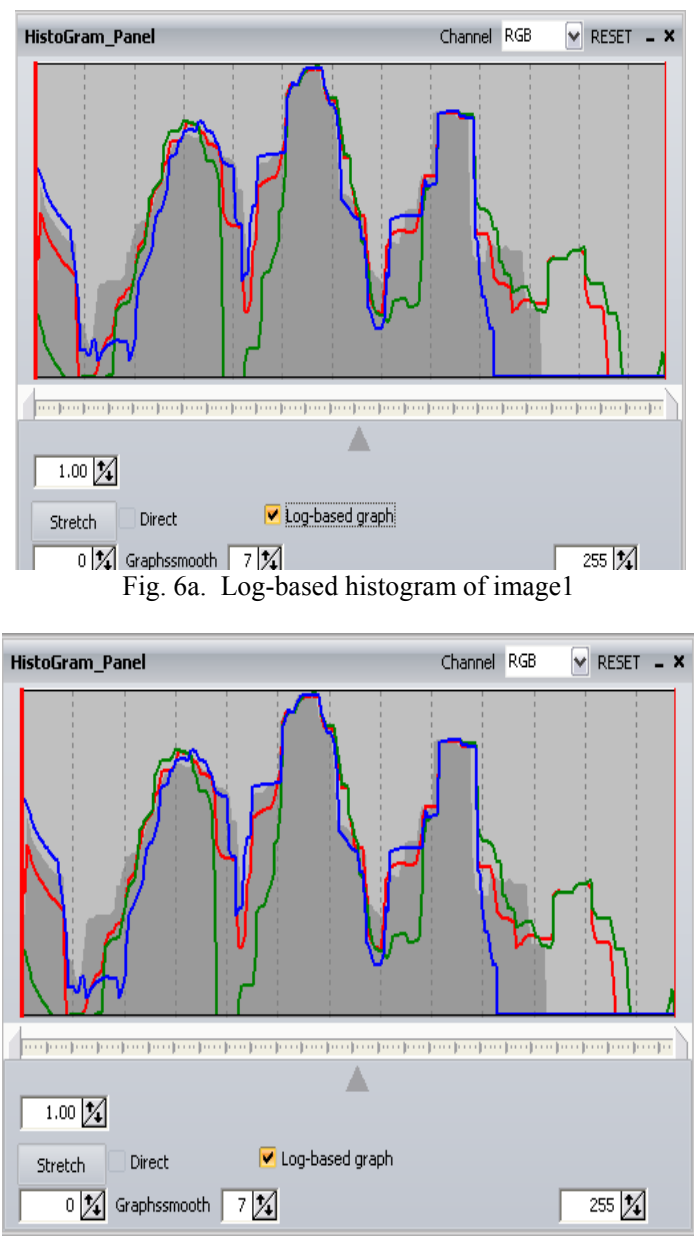

Fig. 6.b Log-based histogram of image2

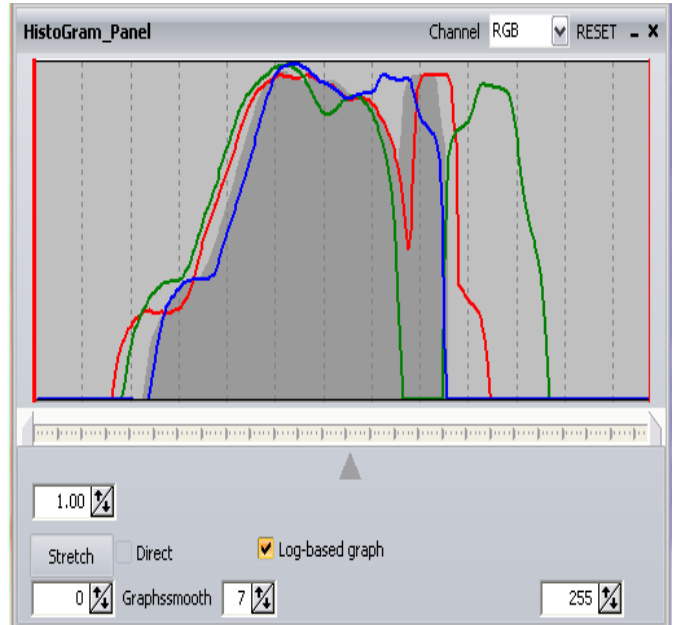

Fig. 6c. Log-based histogram of fused image

\section{Singular Value Decomposition (SVD) Analysis}

Singular Value Decomposition (SVD) is said to be a significant topic in linear algebra by many renowned mathematicians. SVD has many practical and theoretical values, other than image compression. One special feature of SVD is that it can be performed on any real $(m, n)$ matrix. It factors A into three matrices $U, S, V$, such that, $A=U S V T$. Where $\mathrm{U}$ and $\mathrm{V}$ are orthogonal matrices and $\mathrm{S}$ is a diagonal matrix. The purpose of (SVD) is to factor matrix A into USV $\mathrm{T}$. The matrix $U$ contains the left singular vectors, the matrix $\mathrm{V}$ contains the right singular vectors, and the diagonal matrix $\mathrm{S}$ contains the singular values. Where the singular values are 
arranged on the main diagonal in such an order

$\sigma 1 \geq \sigma 2 \geq \ldots \geq \sigma \mathrm{r}>\sigma \mathrm{r}+1=\ldots \sigma \mathrm{p}=0$,

where $r$ is the rank of matrix A, and where (p) is the smaller of the dimensions $\mathrm{m}$ or $\mathrm{n}[16]$.
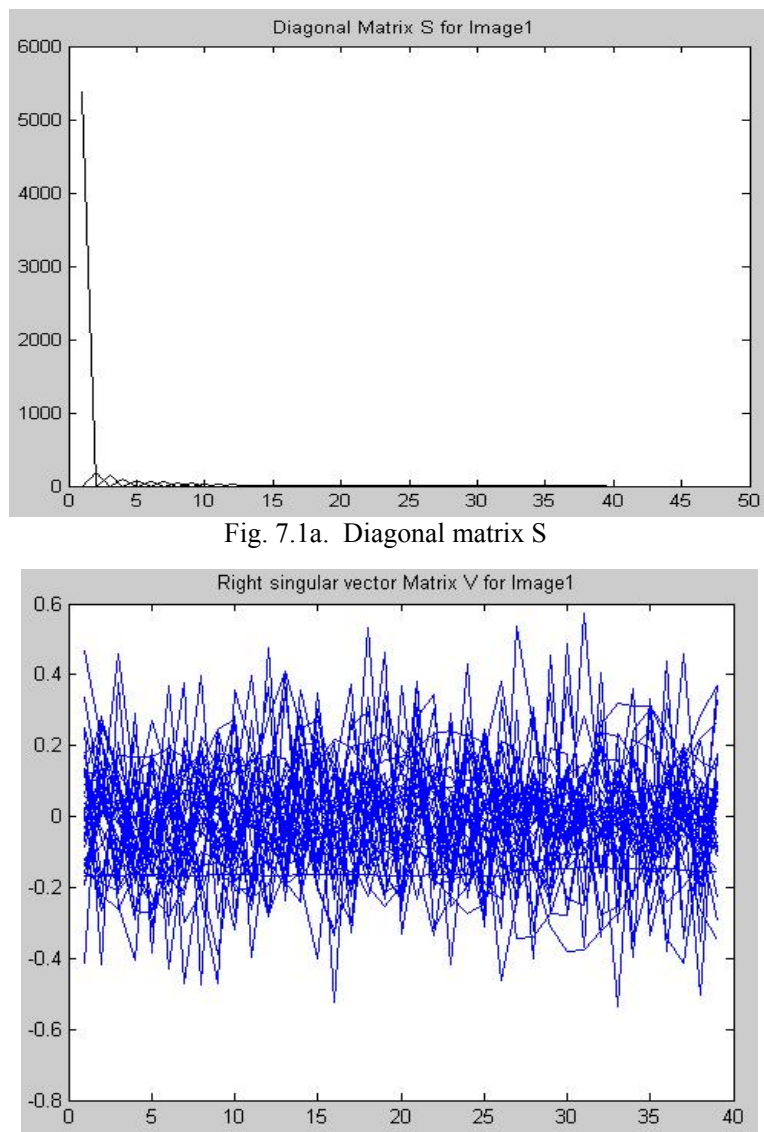

Fig. 7.1b. Right singular vector matrix $\mathrm{V}$

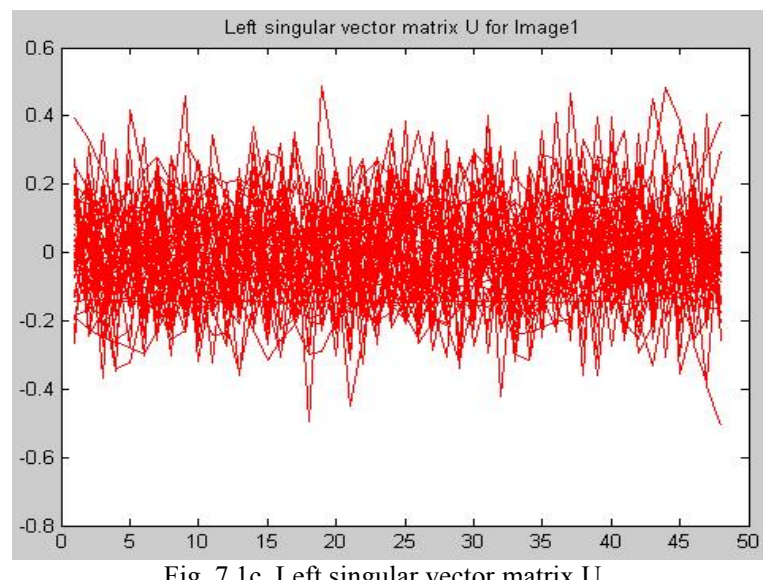

Fig. 7.1c. Left singular vector matrix $U$

\section{A. The Energy of an Image}

In many cases, it is useful to associate energy to an image. If we want to compress an image, we usually want to remove as much data as possible without losing too much energy of the image. The difference in energy between the original image and the compressed image is one possible way to design an error measure when compressing images. However, it is important to notice that this measure is not always appropriate as an error measure. In some cases, the energy error measure might be small, while the visual error is terrible. One difficult problem in image processing is to find a good way of measuring error so that the error measure "agrees with our eyes".
Let a digital image be represented as the matrix I and denote its elements as $\mathrm{m} \times \mathrm{n}$ matrix I and denote its elements as Iij , $i=1,2,3, \ldots m$ and $j=1,2,3, \ldots n$. We define the frobenius norm [17] of the matrix I as

$$
\|I\|_{\text {Fro }}=\sqrt{\sum_{i=1}^{m} \sum_{j=1}^{n}\left|I_{i j}\right|^{2}} .
$$

This is not the most common matrix norm, and not always very convenient for evaluation, moreover it is beyond the scope of the paper, the point to discuss is the analysis of SVD, in which Kristian Sandberg [18] has shown with exercises of various images that the singular value decay is related with the energy of the image. For the images with less information content, the elements of diagonal matrix $S$ reaches peak value and decays gradually as compared with images with more information content whose peak is more than the former and decay is prompt. As shown in Fig.7, the singular value decay of fused image varies with the other two images, thus signifying more energy associated with the fused image.
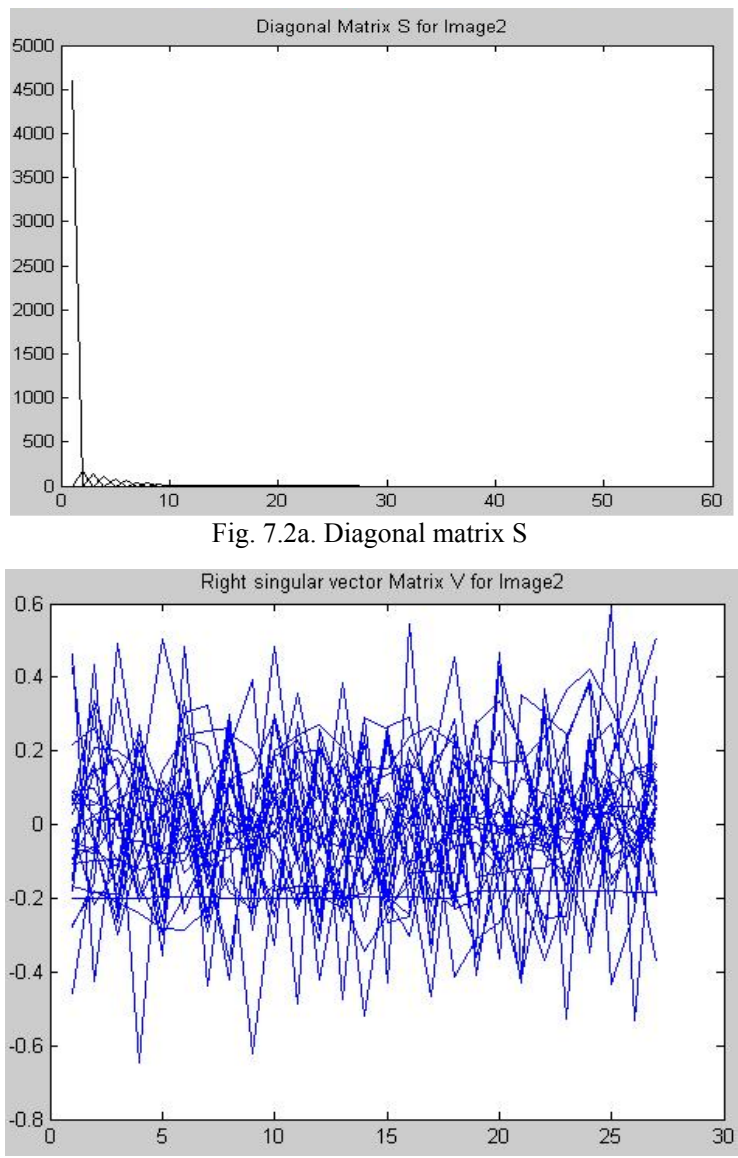

Fig. 7.2b. Right singular vector matrix V

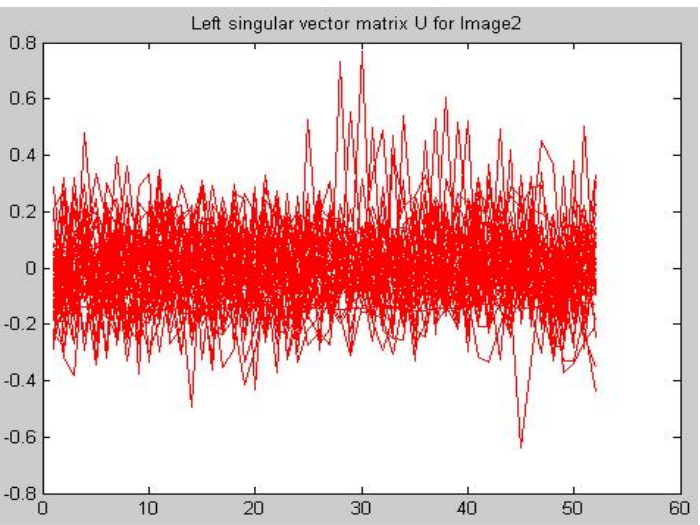

Fig. 7.2c. Left singular vector matrix $U$ 


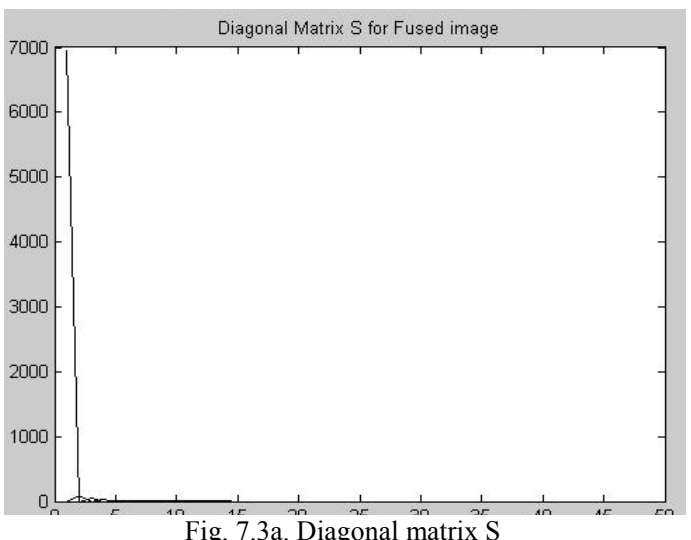

Fig. 7.3a. Diagonal matrix S

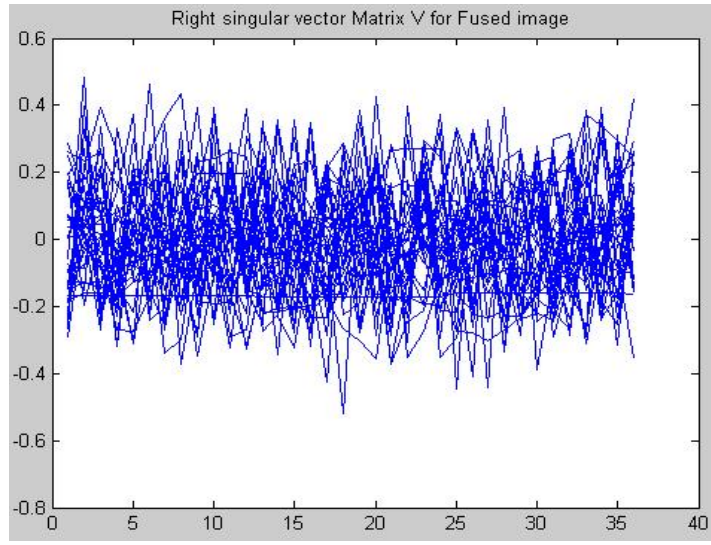

Fig. 7.3b. Right singular vector matrix V

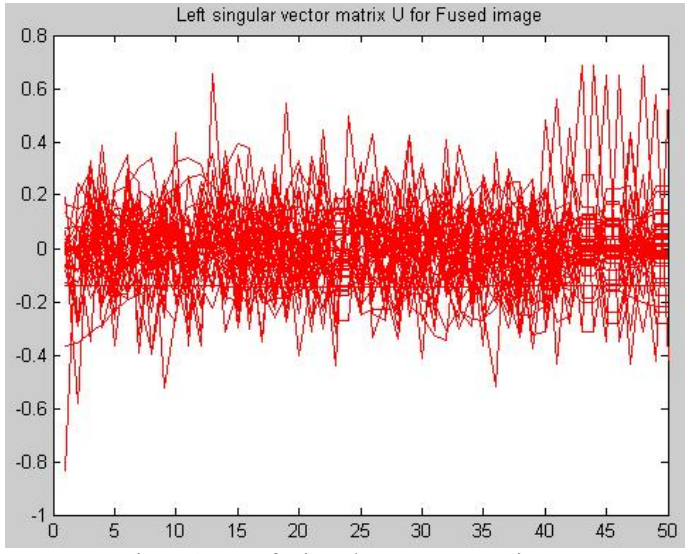

Fig. 7.3c. Left singular vector matrix U

\section{CONCLUSION}

Multisensor data fusion is establishing significant advantages in various engineering applications by improving accuracy and precision in evaluation process. The data in this experiment is considered as image data, captured from image sensor. A simple demonstration was carried out to explore the virtue of data fusion to improve the quality of image. Thus, by processing and analyzing the images, it is shown that information content in the fused image is more than individual image. The experiment also tries to explore use of low cost, moderate resolution camera to render better quality images using data fusion technique.

\section{REFERENCES}

[1] J. Llinas and D. L. Hall, "An introduction to multi-sensor data fusion," in Proc. IEEE International Symposium on Circuits and Systems, May, Monterey, USA, 1998, pp. 537-540.

[2] O. Sidek and S. A. Quadri, "A review of data fusion models and systems," International Journal of Image and Data Fusion, vol. 3, no. 1, pp. 3-21, Jan. 2012

[3] H. B. Mitchell, "Image Fusion: Theories, Techniques and Applications," 1 st ed. Berlin Heidelberg: Springer-Verlag, 2008, pp $1-8$.

[4] G. D. Jones, R. E. Allsop, and J. H. Gilby, "Bayesian analysis for fusion of data from disparate imaging systems for surveillance," Image and Vision Computing, vol. 21, pp. 843-849, 2003.

[5] E. P. Bennett, L. Mason, and M. Millan, "Multispectral bilateral video fusion," IEEE Trans. on Image Processing, vol. 16, pp.1185-1194, 2000 .

[6] M. Mignotte, "Segmentation by fusion of histogram-based K -means clusters in different color spaces," IEEE Trans. on Image Processing, vol. 17, pp. 780-787, 2008.

[7] Y. Zhang, "Methods for image fusion quality assessment- A review, comparison and analysis," in Proc. International archives of the photogrammetric, remote sensing and spatial information sciences, vol. XXXVIII, part B7, Beijing, 2000, pp. 45-56.

[8] A. G. Klein. Data fusion to improve image quality for visual analysis [Online]. Available: http://geography.tamu.edu/class/aklein/geog661/lecture_notes/lecture 08/data_fusion.pdf

[9] O. Sidek, S. A. Quadri, and S. Kabir, "Data fusion technique to improve image quality of low-resolution optical devices," Journal of Electron Devices, vol. 10, pp. 506-510, 2011.

[10] T. Massey, R. Kapur, F. Dabiri, L. N. Vu, and M. Sarrafzadeh, "Localization Using Low-Resolution Optical Sensors," in Proc. fourth IEEE Int. Conf. Mobile Ad-hoc and Sensor Systems, Pisa, Italy, 2007, pp. 1-9.

[11] W. Bressers, "Real-time face verification using a low-resolution camera," BSc Dissertation, Dept.Elect.Eng., University van Amsterdam, Netherlands, 2009.

[12] P. H. H. Yeomans, S. Baker, and B. V. Kumar, "Simultaneous super-resolution and feature extraction for recognition of low resolution faces," in Proc. IEEE Conference on Computer Vision and Pattern Recognition, Anchorage, Alaska, USA ,2008, pp. 1-8.

[13] L. S. Woong, J. Park, and L. S. Whan, "Face reconstruction with low-resolution facial images by feature vector projection in kernel space”, in Proc. 18th Int. Conf. on Pattern Recognition, Hong Kong, China, 2006, pp.1179- 1182 .

[14] Dream Fusion software. [Online]. Available: http://dreamfusion.downloadpipe.com/

[15] MATLAB Simulink. [Online]. Available: www.mathworks.com.

[16] A. Adam and R. David. Image Compression using Singular Value Decomposition. [Online]. Available: http://online.redwoods.cc.ca.us/instruct/darnold/laproj/fall2001/adam dave/textwriteup.pdf

[17] D. Kalman, "A Singularly Valuable Decomposition: The SVD of a Matrix," The College Mathematics Journal, vol. 27, 1996, pp.12-19.

[18] K. Sandberg. The Singular Value Decomposition of an Image. [Online]. http://amath.colorado.edu/courses/5720/2000Spr/Labs/SVD/

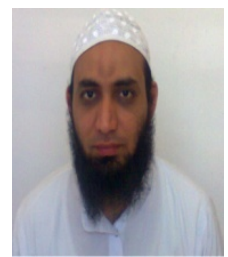

S. A. Quadri is associated with Collaborative Micro-electronic Design Excellence Centre (CEDEC). He graduated B.E (Electronics and Communication) from Karnataka University Dharwad and M.Tech (Computer science \& Engineering) from Visvesvaraya Technological University (VTU), India. His research domain includes neural networks \& multisensor data fusion in various engineering applications.

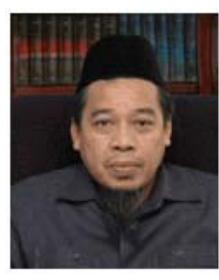

Othman bin Sidek an esteemed Malaysian scientist graduated as Bachelor if Applied Science in electronics (Honors) from Universiti Sains Malaysia and went on to earn a Master of Science Degree in Communication engineering from UMIST, UK, as well as a $\mathrm{PhD}$ in Information Systems Engineering from Bradford University, UK. He is also the Founder and Director of the Collaborative Micro-electronic Design Excellence Centre (CEDEC), an RM 23.7 million approved centre at Universiti Sains Malaysia by the Malaysian Ministry of Finance in 2005 out of his own initiative. His current research areas mainly focus on Micro-Electro Mechanical Systems (MEMS) and Wireless Sensor Network, Embedded System/SOC and VLSI/ASIC Design. All these are the focused areas of CEDEC. 